Check for updates

Cite this: J. Mater. Chem. B, 2021, 9, 1888

\section{A pH/redox-dual responsive, nanoemulsion- embedded hydrogel for efficient oral delivery and controlled intestinal release of magnesium ions $\dagger$}

\author{
Yu Huang, $\ddagger^{\mathrm{a}}$ Zewei Wang, $\ddagger^{\mathrm{a}}$ Guiju Zhang, (D) ab Jie Ren, ${ }^{a}$ Li Yu, ${ }^{a}$ Xuhan Liu, ${ }^{a}$ \\ Yuanxi Yang, ${ }^{a}$ Abirami Ravindran, ${ }^{a}$ Chloe Wong ${ }^{a}$ and Rongjun Chen (D) *a
}

\begin{abstract}
It remains a major challenge to achieve efficient oral delivery and controlled intestinal release of ions using hydrogels. Herein, we report a novel, pH/redox-dual responsive, nanoemulsion-embedded composite hydrogel to address this issue. The hydrogel was first synthesized by crosslinking a biocompatible, $\mathrm{pH}$ responsive pseudopeptide, poly(L-lysine isophthalamide) (PLP), and redox-sensitive L-cystine dimethyl ester dihydrochloride (CDE). A suitable amount of magnesium acetate was encapsulated into oil-in-water nanoemulsions, which were then embedded into the lysine-based hydrogel. The resulting composite hydrogel collapsed into a compact structure at acidic gastric $\mathrm{pH}$, but became highly swollen or degraded in the neutral and reducing intestinal environment. The ion release profiles indicated that the nanoemulsion-embedded composite hydrogel could well retain and protect magnesium ions in the simulated gastric fluid (SGF) buffer at $\mathrm{pH}$ 1.2, but efficiently release them in the simulated intestinal fluid (SIF) buffer at $\mathrm{pH} 6.8$ in the presence of 1,4-dithiothreitol (DTT) as a reducing agent. Moreover, this composite hydrogel system displayed good biocompatibility. These results suggested that the $\mathrm{pH} / \mathrm{redox}$-dual responsive, nanoemulsion-embedded composite hydrogel could be a promising candidate for efficient oral delivery and controlled intestinal release of magnesium and other ions.
\end{abstract}

Received 15th October 2020, Accepted 26th January 2021 DOI: $10.1039 / d 0 t b 02442 b$

rsc.li/materials-b can cause hypermagnesemia, especially in patients with reduced renal function, which results in a number of side effects, such as hypotension, cardiac arrest and neuromuscular disorder. ${ }^{6-8}$ Thus, there is growing interest to introduce a suitable amount of magnesium ions through an alternative delivery route, in particular oral administration that is widely used due to its convenience, pain avoidance and high patient compliance. ${ }^{9}$

Hydrogels are three-dimensional crosslinked polymer networks, which can be extensively swollen in water. ${ }^{10,11}$ Due to their high-water content, hydrogels are considered to have high biocompatibility and have been widely used in drug delivery and tissue engineering. ${ }^{12-14}$ Despite continuous advancements in the development of hydrogels for oral delivery of hydrophilic/ hydrophobic drugs, the use of hydrogels in ion delivery remains a major challenge. This is because their inherent hydrophilic nature and large pore size can prevent stable retainment of ultra-small ions in hydrogel matrices, leading to the unwanted drug leakage and dose dumping. ${ }^{15-17}$ Also importantly, the release profile of loaded ions in most hydrogels can hardly be controlled, which in turn usually causes an hypermagnesemia. ${ }^{18,19}$ Therefore, an efficient hydrogel delivery platform that can stably retain magnesium ions during the delivery but enable controlled release at a target site is needed for treatment of magnesium deficiency. 


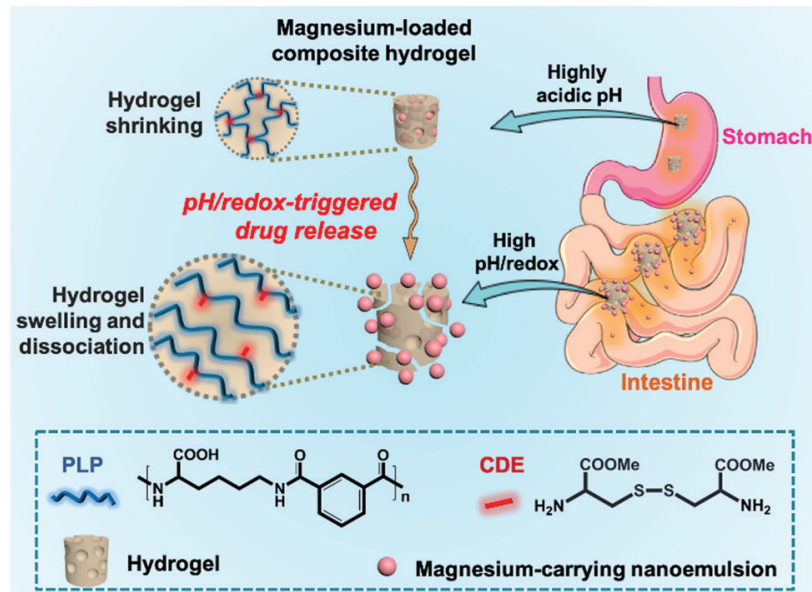

Fig. 1 Schematic illustration of the $\mathrm{pH} /$ redox-dual responsive, nanoemulsionembedded hydrogel for efficient oral delivery and triggered intestinal release of magnesium ions.

In order to address this issue, one attractive approach is to develop composite hydrogels with embedded nanoparticles, which can efficiently carry and retain magnesium ions. Furthermore, composite hydrogels should be designed to respond to stimuli, such as $\mathrm{pH}$ and redox potential changes along the gastrointestinal (GI) tract to achieve controlled drug release. ${ }^{20-24}$ The stomach has a very low pH range of 1-3.0 and limited surface area for drug absorption. On the contrary, the small intestine and colon have a $\mathrm{pH}$ range of 5.5-7.5, high reduction potential, extensive surface area and high drug absorption rates. ${ }^{25-27}$ Stimuli-responsive composite hydrogels are thus required to retain magnesium ions in the stomach and then release in the intestine.

In this work, we report a novel, $\mathrm{pH} /$ redox-dual responsive, nanoemulsion-embedded composite hydrogel for efficient oral delivery and controlled intestinal release of magnesium ions (Fig. 1). A lysine-based, biocompatible hydrogel was previously synthesized from pH-responsive, metabolite-derived poly(L-lysine isophthalamide) (PLP) crosslinked with L-cystine dimethyl ester dihydrochloride (CDE) bearing a disulfide bond for oral drug delivery. ${ }^{28}$ The PLP is a biocompatible, pseudopeptidic polycarboxylate with a $\mathrm{p} K_{\mathrm{a}}$ (negative logarithm of the acid dissociation constant) of around 4.4 and its constituent components, lysine and isophthalic acid, have low toxicity and do not accumulate in the bodily tissues. ${ }^{29-31}$ At an acidic $\mathrm{pH}$, protonation of PLP causes the hydrogel to collapse and form compact structure, thus retaining drugs. At a neutral $\mathrm{pH}$, the carboxylic acid groups pendant to the PLP backbone become negatively charged, leading to swelling of the hydrogel. Further addition of a reducing agent can facilitate degradation of the hydrogel due to the cleavage of disulfide linkages, enabling rapid but triggered-release of drugs in the intestinal environment. ${ }^{24,28,32,33}$ This smart hydrogel with $\mathrm{pH} /$ redox-dual-responsive drug release behavior has an advantage over the previously reported hydrogel based on the coordination bond, which showed the sustained release of bioactive cations, ${ }^{34,35}$ in the application for oral drug delivery. On the other hand, nanoemulsions have been widely utilized for oral delivery of drugs, supplements and nutraceuticals. ${ }^{36-38}$ In the present study, oil-in-water nanoemulsions were fabricated with biocompatible ingredients to accommodate a suitable amount of magnesium acetate, and then embedded into the crosslinked PLP matrices to prepare a composite hydrogel. The physicochemical properties and stability of the magnesium-carrying nanoemulsions were investigated by dynamic light scattering (DLS) and transmission electron microscopy (TEM). Fourier-transform infrared (FTIR) spectroscopy, scanning electron microscopy (SEM) and differential scanning calorimetry (DSC) were employed to characterize the CDE-crosslinked PLP hydrogels. By investigating the swelling properties, magnesium loading efficiencies and $\mathrm{pH} /$ redoxtriggered release profiles, the applicability of the novel nanoemulsion-embedded composite hydrogel as an oral magnesium delivery system was evaluated. Moreover, the cytotoxicity of the nanoemulsion-embedded hydrogel was examined to demonstrate its biocompatibility.

\section{Materials and methods}

\section{Materials}

Kolliphor $^{\circledR}$ RH 40 (polyoxyl 40 hydrogenated castor oil), oleic acid, L-cystine dimethyl ester dihydrochloride (CDE), $N$-hydroxysuccinimide (NHS), $N$-(3-dimethylaminopropyl)- $N^{\prime}$ ethyl carbodiimide hydrochloride (EDC), 1,4-dithiothreitol (DTT), deuterated dimethyl sulfoxide (DMSO- $d_{6}$ ), dimethylformamide (DMF) and anhydrous magnesium acetate $\left(\mathrm{Mg}\left(\mathrm{CH}_{3} \mathrm{COO}\right)_{2}\right)$ were purchased from Sigma-Aldrich (Dorset, UK). Disodium hydrogen phosphate $\left(\mathrm{Na}_{2} \mathrm{HPO}_{4}\right)$, monosodium phosphate $\left(\mathrm{NaH}_{2} \mathrm{PO}_{4}\right)$, sodium hydroxide $(\mathrm{NaOH})$ and hydrochloric acid $(\mathrm{HCl})$ were obtained from VWR International Ltd (Lutterworth, UK). Citric acid, sodium chloride ( $\mathrm{NaCl})$, monopotassium phosphate $\left(\mathrm{KH}_{2} \mathrm{PO}_{4}\right)$ and nitric acid were obtained from Thermo Fisher Scientific (Loughborough, UK).

The simulated gastric fluid (SGF) and simulated intestinal fluid (SIF) were prepared as previously reported. ${ }^{28,39}$ Briefly, the SGF buffer was prepared by adding $0.5 \mathrm{~g} \mathrm{NaCl}$ and $1.75 \mathrm{~mL} \mathrm{HCl}$ into $250 \mathrm{~mL}$ deionized (DI) water, and then the $\mathrm{pH}$ was adjusted to 1.2. The SIF buffer was prepared by adding $1.7 \mathrm{~g} \mathrm{K \textrm {K } _ { 2 }} \mathrm{PO}_{4}$ and $0.225 \mathrm{~g} \mathrm{NaOH}$ into $250 \mathrm{~mL}$ DI water, and the $\mathrm{pH}$ was adjusted to 6.8. The citrate buffer was prepared by adding $0.588 \mathrm{~g}$ citrate powder into $250 \mathrm{~mL}$ DI water $(100 \mathrm{mM})$, and then the $\mathrm{pH}$ was adjusted to 3.0. The concentrated nitric acid was diluted to $2 \%$ (v/v) using DI water before measurement of magnesium by inductively coupled plasma mass spectrometry (ICP-MS).

\section{Synthesis of hydrogels}

The $\mathrm{pH} / \mathrm{redox}$-dual responsive hydrogel was synthesized by an EDC/NHS coupling reaction between PLP and CDE in aqueous solution. Firstly, the polymer PLP was prepared according to the synthesis route shown in Fig. S1 (ESI $\dagger$ ) and its structure was confirmed by a $400 \mathrm{MHz}$ NMR spectrometer (Bruker, Germany) in DMSO- $d_{6}$ (Fig. S2, ESI $\left.\dagger\right)$. The molecular weights of PLP $\left(M_{\mathrm{w}}=\right.$ $\left.35.7 \mathrm{kDa}, M_{\mathrm{n}}=17.9 \mathrm{kDa}, M_{\mathrm{w}} / M_{\mathrm{n}}=1.994\right)$ were measured using an aqueous GPC system (Viscotek, UK) according to the previous publication. ${ }^{40,41}$ Five hydrogels were synthesized with polymer 
concentrations at $5 \mathrm{wt} \%, 7 \mathrm{wt} \%, 9 \mathrm{wt} \%, 13 \mathrm{wt} \%$ and $17 \mathrm{wt} \%$, respectively, with the detailed formulae listed in Table S1 (ESI $\dagger$ ). In a typical procedure, PLP was dissolved in $1 \mathrm{~mL}$ of DI water at a specific concentration in a 24 -well plate ( $14 \mathrm{~mm}$ in well diameter), followed by the addition of $\mathrm{CDE}$ ( 0.2 molar equivalents of the PLP residue) during mixing. EDC ( 2 molar equivalents of the PLP residue) and NHS ( 0.5 molar equivalents of the PLP residue) were then added and the mixture was left overnight at room temperature for gelation. Finally, the synthesized hydrogel was dialyzed for at least 3 days to remove impurities and then stored in DI water.

\section{Characterization of hydrogels}

The FTIR spectra of the lyophilized hydrogels were recorded on a PerkinElmer Spectrum 100 FTIR spectrometer. The differential scanning calorimeter (DSC) was applied to measure thermal properties of the CDE-crosslinked PLP hydrogels by using a TA Instruments DSC Q200 at $10{ }^{\circ} \mathrm{C} \mathrm{min}{ }^{-1}$. The data of the second thermal cycle $\left(80{ }^{\circ} \mathrm{C}\right.$ to $\left.-60{ }^{\circ} \mathrm{C}\right)$ was collected by the TA DSC software. The morphology of the hydrogel was measured by JEOL JSM-5610LV SEM with $20 \mathrm{kV}$.

\section{Preparation of magnesium-carrying nanoemulsions}

The magnesium-carrying nanoemulsions were prepared by selfemulsification. ${ }^{42,43}$ Briefly, an oil phase was made by adding $18.5 \mathrm{~g}$ Kolliphor ${ }^{\circledR} \mathrm{RH} 40$ and $5 \mathrm{~g}$ oleic acid into $76.5 \mathrm{~g}$ ethanol. After complete dissolution, a certain amount of magnesium acetate was added into the oil phase and stirred for $30 \mathrm{~min}$. $20 \mathrm{mM}$ of phosphate buffer saline (PBS, $\mathrm{pH}$ 7.4) was prepared as an aqueous phase. Then, the oil phase was slowly added into the aqueous phase $(1: 1, \mathrm{v} / \mathrm{v})$ under constant stirring at room temperature. After mixing of the oil and aqueous phases, stirring was continued for another $30 \mathrm{~min}$ to ensure complete dispersion. Blank nanoemulsions without magnesium acetate in the oil phase were synthesized as control.

\section{Characterization of magnesium-carrying nanoemulsions}

The hydrodynamic size and polydispersity index (PDI) of the nanoemulsion systems were measured by DLS using a Malvern Zetasizer Nano S, and their zeta potentials were measured using a Brookhaven ZetaPALS Zeta Potential Analyzer. The nanoemulsion morphology was examined via TEM using a JEOL JEM2100F instrument. TEM samples were prepared by dropping the nanoemulsion solution on formvar carbon-coated copper grids. The stability of the nanoemulsion systems was measured by monitoring the changes of DLS size and PDI. The concentrations of magnesium were measured by ICP-MS using an Agilent Technologies 7900 ICP-MS after dilution of the samples in $2 \%$ (v/v) nitric acid. Measurements were repeated in triplicates.

\section{Embedding magnesium-carrying nanoemulsions into hydrogels}

Hydrogel disks were prepared in a 24-well plate $(14 \mathrm{~mm}$ in well diameter) in order to minimize variations in the hydrogel dimension. In a typical procedure of embedding nanoemulsions into the PLP hydrogel, one hydrogel disk was first placed into a $50 \mathrm{~mL}$ of centrifuge tube containing $3 \mathrm{~mL}$ of $100 \mathrm{mM}$ citrate buffer $(\mathrm{pH} 3.0)$ at room temperature for $48 \mathrm{~h}$. After discarding the citrate buffer, the hydrogel disk was rinsed using DI water and then immersed into the $20 \mathrm{~mL}$ of magnesiumcarrying nanoemulsions for nanoemulsion embedding at room temperature for $48 \mathrm{~h}$. After that, $5.4 \mu \mathrm{L}$ of $14 \%(\mathrm{v} / \mathrm{v}) \mathrm{HCl}$ solution was added to shrink the hydrogel for $24 \mathrm{~h}$. Finally, the hydrogel disk was removed from the nanoemulsion solution and rinsed with DI water. The spent nanoemulsion loading solution and washing solution were combined to measure the unloaded magnesium through ICP-MS. The encapsulation efficiency of magnesium in this nanoemulsion-embedded hydrogel was calculated according to the equation below.

$$
\text { Encapsulation efficiency }(\%)=\frac{m_{1}}{m_{\mathrm{t}}} \times 100
$$

where $m_{1}$ is the weight of magnesium encapsulated into the hydrogel, $m_{\mathrm{t}}$ is the total weight of magnesium initially in the nanoemulsion embedding solution. Measurements were repeated in quintuplicate.

\section{Swelling behavior}

The hydrogel disks were placed in $100 \mathrm{mM}$ phosphate buffer (pH 7.4) at room temperature for $48 \mathrm{~h}$, and then moved to three different buffer solutions for $6 \mathrm{~h}$ in a water bath at $37{ }^{\circ} \mathrm{C}$ with gentle agitation: (1) SGF at pH 1.2, (2) SIF at pH 6.8 and (3) SIF in the presence of $125 \mathrm{mM}$ of DTT at $\mathrm{pH}$ 6.8. After that, their wet weights $\left(m_{\mathrm{w}}\right)$ were measured. These samples were lyophilized using a VirTis BenchTop Pro freeze-dryer to obtain their dry weights $\left(m_{\mathrm{d}}\right)$. The swelling ratio $(q)$ was calculated according to the following equation. Measurements were repeated in quadruplicate.

$$
\text { Swelling ratio }(q)=\frac{m_{\mathrm{w}}}{m_{\mathrm{d}}}
$$

\section{Magnesium release study}

The magnesium-carrying nanoemulsion-embedded hydrogel disks were placed into a tube containing $10 \mathrm{~mL}$ of specific biorelevant release buffers, such as (1) SGF at pH 1.2, (2) SIF at pH 6.8 and (3) SIF in the presence of $125 \mathrm{mM}$ of DTT at pH 6.8, respectively, and then incubated in a water bath at $37{ }^{\circ} \mathrm{C}$ with gentle agitation. At specific time intervals, $50 \mu \mathrm{L}$ of samples were taken out of each tube and replaced with $50 \mu \mathrm{L}$ of the respective fresh buffers. The $50 \mu \mathrm{L}$ of samples were diluted with $2 \%(\mathrm{v} / \mathrm{v})$ nitric acid to $5 \mathrm{~mL}$ before magnesium measurements by ICP-MS. The percentage of released magnesium was calculated according to the following equation:

$$
\text { Magnesium release }(\%)=\frac{m_{\mathrm{r}}}{m_{\mathrm{l}}} \times 100
$$

where $m_{\mathrm{r}}$ is the amount of released magnesium at a specific time point and $m_{1}$ is the amount of magnesium loaded into the hydrogels. Measurements were repeated in triplicate.

\section{Cytotoxicity study}

The cytotoxicity of the blank PLP-CDE hydrogel and the nanoemulsions with and without magnesium toward human cervical 
carcinoma HeLa cells was evaluated by Alamar Blue assay. Briefly, HeLa cells were cultured in Dulbecco's Modified Eagle's Medium (DMEM) supplemented with 10\% (v/v) fetal bovine serum (FBS), penicillin (100 units $\mathrm{mL}^{-1}$ ) and streptomycin $\left(100 \mu \mathrm{g} \mathrm{mL}^{-1}\right)$ and incubated in a humidified incubator with $5 \% \mathrm{CO}_{2}$ at $37{ }^{\circ} \mathrm{C}$. HeLa cells were seeded into 96-well plates at a density of $8 \times 10^{3}$ cells per well in $200 \mu \mathrm{L}$ of DMEM and cultured until $70-80 \%$ confluence. After an overnight incubation, the spent medium was carefully removed and replenished with $200 \mu \mathrm{L}$ of fresh DMEM containing the PLP-CDE hydrogel, blank nanoemulsions and magnesium-carrying nanoemulsions at various concentrations respectively. The cells without sample treatment were used as control. After $24 \mathrm{~h}$ of incubation, cells were washed with PBS, and incubated with the DMEM containing $10 \%(\mathrm{v} / \mathrm{v})$ of alamarBlue for $4 \mathrm{~h}$ of further incubation. The fluorescence of each well was determined using a Promega GloMax ${ }^{\circledR}$-Multi+ Microplate Multimode Reader at the excitation wavelength of $525 \mathrm{~nm}$ and emission wavelengths of 580-640 nm. The wells with alamarBlue but without cells were used as background. Cell viabilities were calculated according to the following equation:

$$
\text { Cell viability }(\%)=\frac{F_{\mathrm{s}}-F_{\mathrm{b}}}{F_{\mathrm{c}}-F_{\mathrm{b}}} \times 100
$$

where $F_{\mathrm{s}}, F_{\mathrm{b}}$ and $F_{\mathrm{c}}$ represent the fluorescence of samples, background and control, respectively. Measurements were repeated in quintuplicate.

\section{Results and discussion}

\section{pH/redox-dual responsive hydrogels}

Hydrogels were prepared by EDC/NHS coupling of PLP and CDE at room temperature (Fig. 2A) in a 24 -well plate (14 $\mathrm{mm}$ in well diameter) to minimize variations in the hydrogel dimension. Five hydrogels, denoted as Gel1, Gel2, Gel3, Gel4 and Gel5 were synthesized with different PLP concentrations at $5 \mathrm{wt} \%, 7 \mathrm{wt} \%$, $9 \mathrm{wt} \%, 13 \mathrm{wt} \%$ and $17 \mathrm{wt} \%$, respectively (Table S1, ESI $\dagger$ ). The FTIR spectrum (Fig. S3, ESI $\dagger$ ) shows the characteristic peaks of the hydrogels at $3298 \mathrm{~cm}^{-1}$ (w, $\nu_{\mathrm{s}} \mathrm{O}-\mathrm{H}$ of carboxylic acid groups), 2935 and $2862 \mathrm{~cm}^{-1}$ (s, $\left.\nu_{\mathrm{s}} \mathrm{C}-\mathrm{H}\right), 1727 \mathrm{~cm}^{-1}\left(\mathrm{~s}, \nu_{\mathrm{s}} \mathrm{C}=\mathrm{O}\right.$ of carboxylic acid groups), $1635 \mathrm{~cm}^{-1}$ (s, Amide I), $1523 \mathrm{~cm}^{-1}$ (s, Amide II), $1435 \mathrm{~cm}^{-1}$ (s, $\left.\delta \mathrm{C}-\mathrm{H}\right)$ and $1266 \mathrm{~cm}^{-1}$ (w, Amide III), which were consistent with our previously reported result. ${ }^{28}$ The DSC spectrum in Fig. S4 (ESI $\dagger$ ) displays a typical thermal cycle of the hydrogels from 80 to $-60{ }^{\circ} \mathrm{C}$. No obvious endothermic peak was detected, suggesting that chemical crosslinks between PLP and CDE could inhibit the crystallization behavior and increase the thermal stability of hydrogels.

The swelling experiments were carried out in SGF ( $\mathrm{pH} 1.2$ ), SIF (pH 6.8) and SIF ( $\mathrm{pH} 6.8)+125 \mathrm{mM}$ of DTT buffers to investigate the $\mathrm{pH}$ and redox responsiveness of the hydrogels. As shown in Fig. S5 (ESI $\dagger$ ) and Fig. 2B, the swelling ratio $q$ of all hydrogels increased as $\mathrm{pH}$ increased from 1.2 to 6.8 after treatment for $1 \mathrm{~h}$ and $6 \mathrm{~h}$, respectively. This was due to the deprotonation of carboxylic acid groups pendant onto the PLP backbone at high $\mathrm{pH}$, which caused the electrostatic repulsion
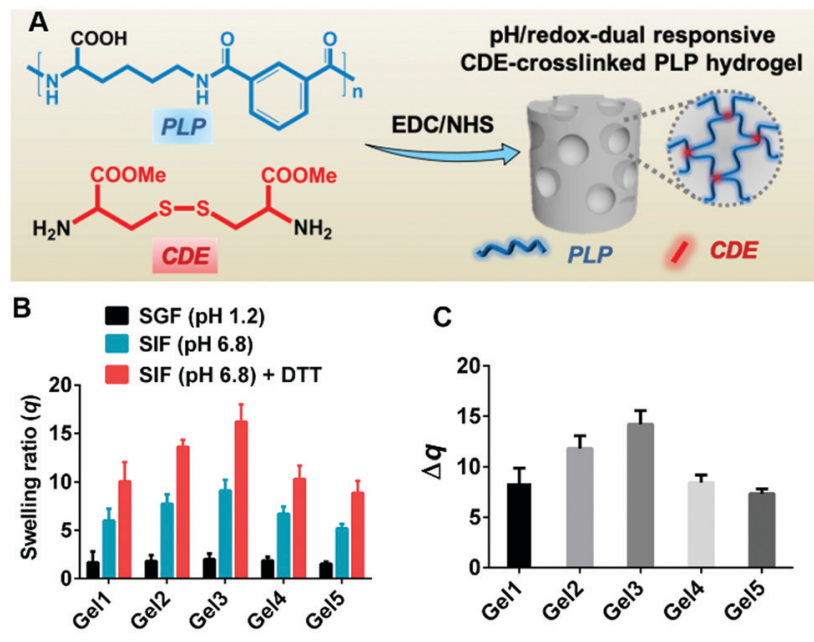

C
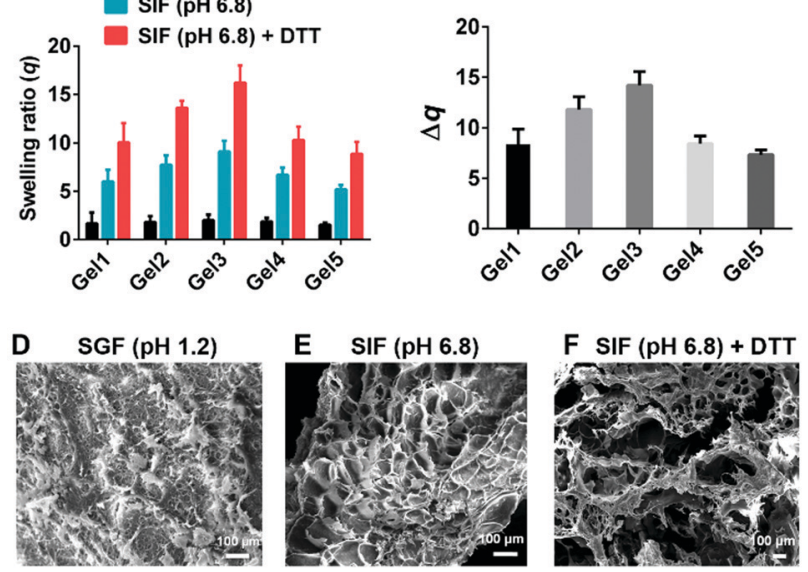

Fig. 2 (A) Schematic illustration of the synthesis of the $\mathrm{pH} /$ redox-dual responsive CDE-crosslinked PLP hydrogel. (B) Swelling ratios $q$ of different hydrogels with the PLP concentration at $5 \mathrm{wt} \%$ (Gel1), $7 \mathrm{wt} \%$ (Gel2), $9 \mathrm{wt} \%$ (Gel3), $13 \mathrm{wt} \%$ (Gel4) and $17 \mathrm{wt} \%$ (Gel5) after treatment with SGF (pH 1.2), SIF (pH 6.8) and SIF (pH 6.8) +125 mM of DTT for 6 h, respectively. (C) The differences in swelling ratio $(\Delta q)$ of different hydrogels between treatments in SGF (pH 1.2) and SIF (pH 6.8) + $125 \mathrm{mM}$ of DTT for $6 \mathrm{~h}$. Data are presented as mean values with standard deviations (mean $\pm S D, n=4$ ). SEM images of Gel3 treated with (D) SGF (pH 1.2), (E) SIF ( $\mathrm{pH}$ 6.8) and (F) SIF $(\mathrm{pH} 6.8)+125 \mathrm{mM}$ of DTT. Scale bar $100 \mu \mathrm{m}$

leading to hydrogel swelling. ${ }^{39}$ In the presence of $125 \mathrm{mM}$ of DTT as a reducing agent, the hydrogels became more swollen, which could be ascribed to the partial cleavage of disulfide bonds in hydrogels after $6 \mathrm{~h}$ of treatment. An extended treatment up to $12 \mathrm{~h}$ with $125 \mathrm{mM}$ of DTT was found to cause complete dissociation of the hydrogels. The swelling ratio gradually increased with increasing the PLP concentration from $5 \mathrm{wt} \%$ to $9 \mathrm{wt} \%$, which might be due to the inadequate gelation when the PLP concentration was below $9 \mathrm{wt} \%$. It was also found that the hydrogel could not be formed when the PLP concentration was no more than $2 \mathrm{wt} \%$. Interestingly, the swelling ratio decreased significantly with increasing the PLP concentration from $9 \mathrm{wt} \%$ to $17 \mathrm{wt} \%$, which indicated that when the polymer concentration was above a threshold for adequate gelation, a lower density of the polymer network could hold more water. As shown in Fig. S6 (ESI $\dagger$ ) and Fig. 2C, among all hydrogels, Gel3 had the largest change in the swelling ratio between the treatment in SGF (pH 1.2) and SIF (pH 6.8) + $125 \mathrm{mM}$ of DTT for $1 \mathrm{~h}$ and $6 \mathrm{~h}$, respectively. The $\Delta q$ after $6 \mathrm{~h}$ of treatment was $13.78 \pm 0.92$. This suggests that Gel3 could retain the payload in its collapsed structure $(q=2.20 \pm 0.56)$ in the highly acidic stomach environment, whilst exhibit efficient payload release in the neutral and reducing intestinal environment via considerable 
hydrogel swelling $(q=15.98 \pm 1.78)$. Thus, Gel3 synthesized with the polymer concentration of $9 \mathrm{wt} \%$ was chosen to prepare the nanoemulsion-embedded composite hydrogel.

The $\mathrm{pH} /$ redox-dual responsive properties of hydrogels were further confirmed by SEM. When Gel3 hydrogels were equilibrated in SGF (pH 1.2), SIF (pH 6.8) and SIF (pH 6.8) + $125 \mathrm{mM}$ of DTT for $6 \mathrm{~h}$, respectively, the porous hydrogel structure varied significantly. As shown in Fig. 2D-F, the hydrogel in SGF (pH 1.2) collapsed to form a compact structure. By comparison, the hydrogel in SIF (pH 6.8) was swollen, leading to much bigger pore sizes. After treatment with SIF ( $\mathrm{pH}$ 6.8) containing the reducing agent DTT, the hydrogel was further swollen and some porous structures were damaged as result of disulfide bond cleavage.

\section{Magnesium-carrying nanoemulsions}

Magnesium acetate was dissolved in the ethanolic solution containing 5 wt\% oleic acid and 18.5 wt\% Kolliphor ${ }^{\circledR}$ RH 40 as an oil phase. As displayed in Fig. 3A, the magnesium-carrying nanoemulsions (400 $\mathrm{mg} \mathrm{mL} \mathrm{mL}^{-1}$ of magnesium acetate) were formed by slowly adding the resulting magnesium-containing oil phase into PBS buffer (20 mM, pH 7.4) as an aqueous phase $(1: 1, \mathrm{v} / \mathrm{v})$ under constant stirring at room temperature. ${ }^{42,43}$ As shown in Fig. 3B and Fig. S7 (ESI $\dagger$ ), the DLS size of magnesiumcarrying nanoemulsions $(23.98 \pm 0.56 \mathrm{~nm})$ was similar to that of
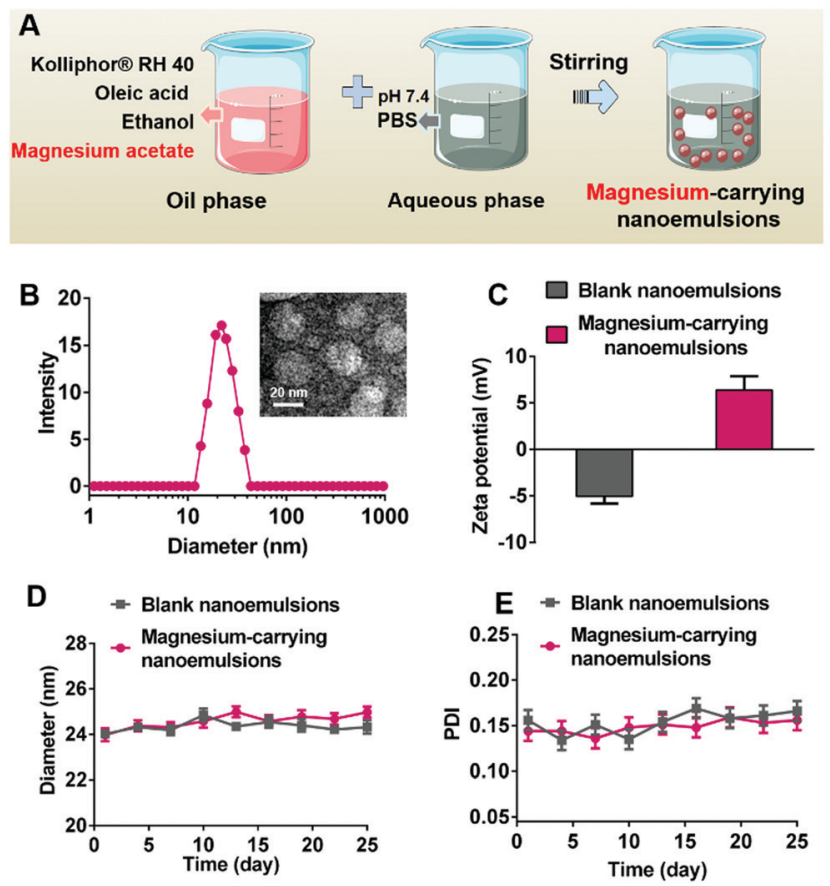

Fig. 3 (A) Schematic illustration of the preparation procedure of magnesiumcarrying nanoemulsions. (B) DLS size distribution of magnesium-carrying nanoemulsions in $\mathrm{pH} 7.4$ PBS buffer at a magnesium acetate concentration of $400 \mathrm{mg} \mathrm{mL}^{-1}$. Inset: TEM image of magnesium-carrying nanoemulsions. Scale bar $20 \mathrm{~nm}$. (C) Zeta potential of blank nanoemulsions and magnesiumcarrying nanoemulsions (400 $\mathrm{mg} \mathrm{mL}^{-1}$ of magnesium acetate) in $\mathrm{pH}$ 7.4 PBS buffer. Time-dependent (D) DLS size and (E) PDI of blank nanoemulsions and magnesium-carrying nanoemulsions ( $400 \mathrm{mg} \mathrm{mL}^{-1}$ of magnesium acetate) in $\mathrm{pH}$ 7.4 PBS buffer at room temperature. Data are presented as mean \pm SD $(n=3)$. blank nanoemulsions $(24.12 \pm 0.38 \mathrm{~nm})$, suggesting that encapsulation of magnesium ions in the nanoemulsions caused a negligible size change. The TEM image confirmed the spherical structure and uniform size of magnesium-carrying nanoemulsions, and the particle size measured by TEM $(22.15 \pm 0.17 \mathrm{~nm})$ was consistent with the DLS result. Fig. 3C showed that blank nanoemulsions were slightly negatively charged $(-5.04 \pm 0.78 \mathrm{mV})$ due to the presence of oleic acid in the oil phase. ${ }^{44}$ The loading of magnesium ions enabled the nanoemulsions to be positively charged with a zeta potential of $6.39 \pm 1.49 \mathrm{mV}$, indicative of successful magnesium encapsulation. Good stability of the nanoemulsions was demonstrated since there were no significant changes in the particle size or polydispersity index (PDI) for both blank nanoemulsions and magnesium-carrying nanoemulsions during storage at room temperature for 25 days (Fig. 3D and E).

\section{Nanoemulsion-embedded hydrogels}

The magnesium-carrying nanoemulsions were embedded into the $\mathrm{pH} /$ redox-dual responsive PLP hydrogel according to the method illustrated in Fig. 4A. As compared to the blank PLP hydrogel, the FTIR spectrum of the composite hydrogel displayed a new broad peak at 1028-1045 $\mathrm{cm}^{-1}$ corresponding to the out-of-plane and in-plane $\mathrm{CH}_{3}$ rocking mode of the $\mathrm{CH}_{3} \mathrm{COO}^{-}$ions, and the vibration band at $950 \mathrm{~cm}^{-1}$ attributing to the $\mathrm{C}-\mathrm{C}$ stretching mode were observed (Fig. S3, ESI $\dagger$ ). These characteristic peaks indicate that magnesium acetate, $\mathrm{Mg}\left(\mathrm{CH}_{3} \mathrm{COO}\right)_{2}$, was successfully encapsulated into the hydrogel. ${ }^{45,46}$ Fig. 4B shows that the amount of magnesium encapsulated in the composite hydrogel disk increased proportionally from $66.4 \pm 6.2$ to $294.7 \pm 4.8 \mathrm{mg}$ with increasing the magnesium acetate concentration of the loading solution of magnesium-carrying nanoemulsions from 100 to $400 \mathrm{mg} \mathrm{mL}^{-1}$. A plateau was reached with further increasing the magnesium acetate concentration. As a result, a maximum magnesium encapsulation efficiency of $32.5 \pm 1.4 \%$ was achieved when magnesium acetate was $400 \mathrm{mg} \mathrm{mL}^{-1}$ in the loading solution,

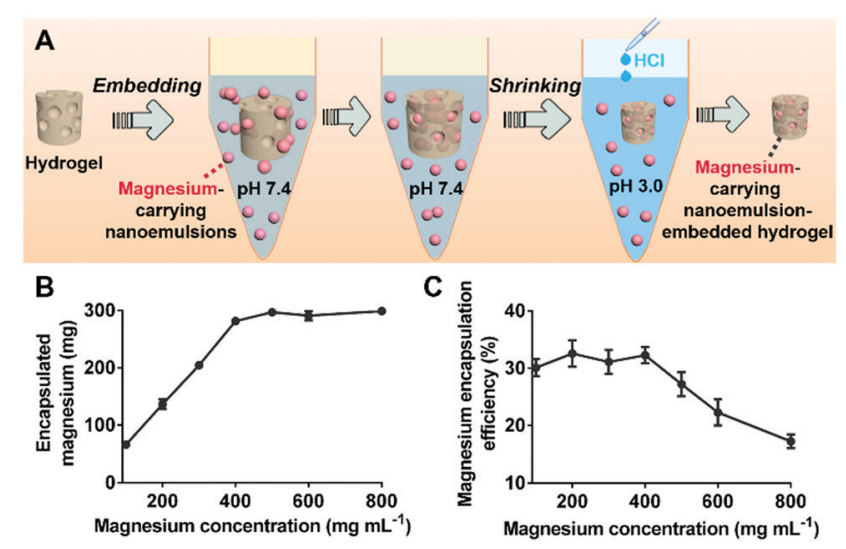

Fig. 4 (A) Schematic illustration of the embedding of magnesium-carrying nanoemulsions into the $\mathrm{pH}$-redox dual-responsive hydrogel. (B) Amount of magnesium encapsulated in the composite hydrogel and (C) magnesium encapsulation efficiency as function of magnesium concentration of the loading solution of magnesium-carrying nanoemulsions. Data are presented as mean \pm SD $(n=5)$. 
whilst a further increase in the magnesium acetate concentration reduced the encapsulation efficiency (Fig. 4C). Thus, the composite hydrogel disk loaded with $294.7 \pm 4.8 \mathrm{mg}$ magnesium was chosen for the further studies herein. This is favorable for oral magnesium delivery because the recommended magnesium dose was approximately $280-355 \mathrm{mg}$ for adult. ${ }^{47}$ In addition, DSC measurements revealed that, like the blank hydrogel (Fig. S4, ESI $\dagger$ ), the magnesium-carrying nanoemulsion-embedded hydrogel system showed no obvious endothermic peak in a typical thermal cycle from 80 to $-60{ }^{\circ} \mathrm{C}$, suggesting that the magnesiumloaded composite hydrogel retained good thermal stability (Fig. $\mathrm{S} 4, \mathrm{ESI} \dagger)$.

\section{Triggered release of magnesium}

The $\mathrm{pH} /$ redox-dual responsiveness and successful magnesium encapsulation of the composite hydrogels prompted us to examine their triggered magnesium release behavior in biorelevant media mimicking the GI tract environment. ${ }^{28,32,33}$ (Fig. 5A). It was found that the magnesium-carrying nanoemulsion-embedded hydrogel collapsed in SGF (pH 1.2), but was dramatically swollen in SIF ( $\mathrm{pH}$ 6.8) in the absence and presence of the reducing agent DTT (Fig. 5B). Fig. S8 (ESI $\dagger$ ) and Fig. 5C show that their swelling ratios $q$ after treatment for $1 \mathrm{~h}$ and $6 \mathrm{~h}$ were similar to the blank hydrogels (Fig. S5, ESI $\dagger$ and Fig. 2B) in SGF $(\mathrm{pH}$ 1.2), SIF (pH 6.8) and SIF ( $\mathrm{pH} 6.8)+125 \mathrm{mM}$ of DTT, respectively, suggesting that embedding of the magnesiumcarrying nanoemulsions did not compromise the $\mathrm{pH} /$ redox-dual responsiveness of the hydrogels.

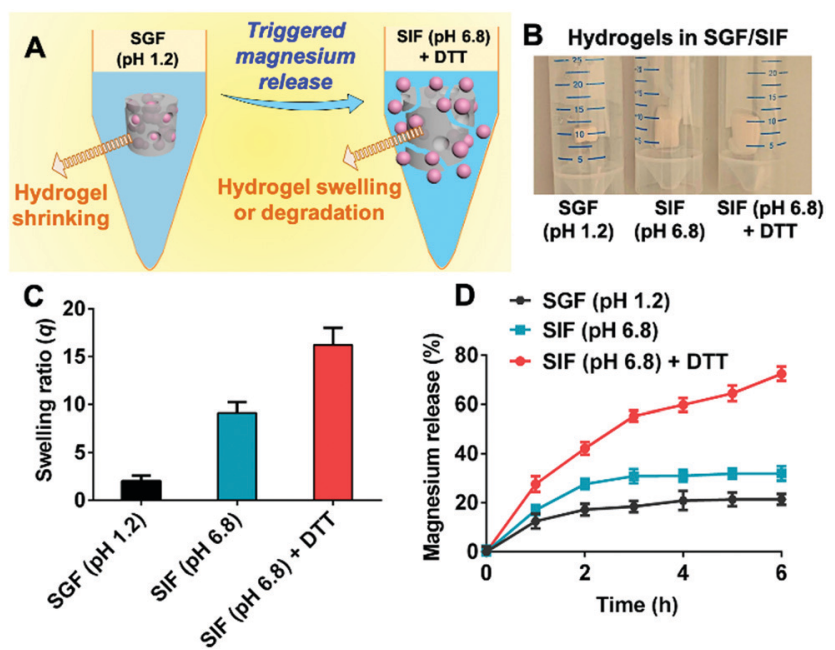

Fig. 5 (A) Schematic illustration of $\mathrm{pH}$ - and redox-triggered release behavior of the magnesium-carrying nanoemulsion-embedded hydrogel. The composite hydrogel could well retain magnesium ions in SGF $(\mathrm{pH}$ 1.2) mimicking the stomach environment, but facilitate efficient release in SIF $(\mathrm{pH} \mathrm{6.8)}+125 \mathrm{mM}$ of DTT mimicking the intestinal environment. (B) Typical photograph of the magnesium-loaded composite hydrogels in SGF (pH 1.2), SIF (pH 6.8) and SIF (pH 6.8) $+125 \mathrm{mM}$ of DTT, respectively. (C) Swelling ratios $q$ of the magnesium-loaded composite hydrogels after treatment of SGF (pH 1.2), SIF (pH 6.8) and SIF (pH 6.8) + $125 \mathrm{mM}$ of DTT buffer for $6 \mathrm{~h}$, respectively. (D) Release kinetics of the magnesium-loaded composite hydrogels in SGF (pH 1.2), SIF (pH 6.8) and SIF (pH 6.8) + $125 \mathrm{mM}$ $\mathrm{DTT}$, respectively. Data presented as mean $\pm \mathrm{SD}(n=3)$.
Fig. 5D shows that the composite hydrogel could successfully retain magnesium ions in the highly acidic gastric environment but efficiently release them in a controlled manner in the intestinal $\mathrm{pH}$ and redox environment. As expected, only a small proportion of the magnesium-carrying nanoemulsions (less than $20.4 \pm 2.2 \%$ magnesium) were released when the composite hydrogel was treated in SGF $(\mathrm{pH}$ 1.2) throughout the period studied. In SIF ( $\mathrm{pH}$ 6.8), the percentage of released magnesium ions (present in the nanoemulsions) reached about $30.8 \pm 2.9 \%$ within first $3 \mathrm{~h}$ and a plateau was then reached afterwards. By comparison, when DTT was added in SIF (pH 6.8) as a redox trigger, the release of magnesium ions (present in the nanoemulsions) continued to increase to $72.5 \pm 2.8 \%$ within $6 \mathrm{~h}$. These results indicate that favorable magnesium release could be efficiently triggered in the neutral and reducing intestinal environment.

It has been well recognized that the size of emulsions is critical for drug bioavailability in oral delivery. ${ }^{48}$ Thanks to the small particle size, nanoemulsions have a large surface area and can therefore interact strongly with biological components along the GI tract. ${ }^{49}$ For example, nanoemulsions $(10-100 \mathrm{~nm})$ have been reported to have a higher digestion rate in the GI tract compared to conventional emulsions since they have more binding sites available for digestive enzymes, such as lipase. ${ }^{50-52}$ Therefore, DLS measurements were carried out to examine if the magnesium-carrying nanoemulsions aggregated or were destroyed post release from the PLP hydrogels treated with SGF (pH 1.2), SIF ( $\mathrm{pH}$ 6.8) and SIF ( $\mathrm{pH}$ 6.8) + $125 \mathrm{mM}$ of DTT, respectively for $6 \mathrm{~h}$. Fig. S9 (ESI $\dagger$ ) show that the particle size (around $23 \mathrm{~nm}$ in diameter) and PDI (around 0.14) of the post-release magnesium-carrying nanoemulsions were similar in all the biorelevant media, remaining almost unchanged compared to their initial size and PDI before being embedded into the hydrogels (Fig. 3B and Fig. S7, ESI $\dagger$ ). The DLS results suggest no aggregation or degradation of the post-release nanoemulsions in the GI tract environment. This implies that these post-release magnesium-carrying nanoemulsions with a small size would confer magnesium ions with good bioavailability in the intestine.

\section{In vitro cytotoxicity}

The Alamar blue assay was carried out to examine the cytotoxicity of blank hydrogels, blank nanoemulsions and magnesium-
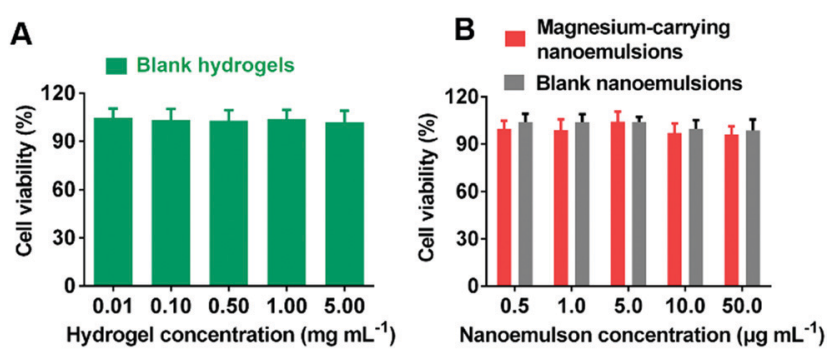

Fig. 6 (A) Concentration-dependent viability of HeLa cells treated with (A) blank hydrogels and (B) blank or magnesium-carrying nanoemulsions, respectively for $24 \mathrm{~h}$ as determined by Alamar Blue assay. Data are presented as mean $\pm \mathrm{SD}(n=5)$. 
carrying nanoemulsions. As shown in Fig. 6, the viabilities of cells were all higher than $95 \%$ within the concentration ranges

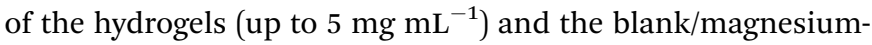
carrying nanoemulsions (up to $50 \mu \mathrm{g} \mathrm{mL}^{-1}$ ) tested, suggesting that the CDE-crosslinked PLP hydrogels and the nanoemulsions had negligible cytotoxicity.

\section{Conclusions}

A pH/redox-dual responsive, nanoemulsion-embedded, CDEcrosslinked composite hydrogel was synthesized. It was demonstrated that at an acidic $\mathrm{pH}$, this hydrogel collapsed and formed a compact structure, while in the neutral and reducing environment, the hydrogel was highly swollen or dissociated. The drug release profiles suggested that the nanoemulsion-embedded composite hydrogel could well retain and protect magnesium ions in the highly acidic gastric environment, but efficiently release them in the neutral and reducing intestinal environment. The high ion encapsulation efficiency, triggered intestinal release and good biocompatibility suggest that this $\mathrm{pH} /$ redox-dual responsive, nanoemulsion-embedded composite hydrogel could be a promising candidate for efficient intestinal delivery of magnesium ions and other ionic therapeutics.

\section{Conflicts of interest}

There are no conflicts to declare.

\section{Acknowledgements}

This research is funded by the Department of Health and Social Care using UK Aid funding and is managed by the Engineering and Physical Sciences Research Council (EPSRC, grant number: EP/R013764/1). The views expressed in this publication are those of the author(s) and not necessarily those of the Department of Health and Social Care. The authors thank Dr Mahmoud Ardakani for the assistance in TEM measurement. The authors would also like to thank Ms Patricia Carry and Mr Kaho Cheung for access to the Analytical Services in the Department of Chemical Engineering at Imperial College London.

\section{Notes and references}

1 J. H. F. De Baaij, J. G. J. Hoenderop and R. J. M. Bindels, Physiol. Rev., 2015, 95, 1-46.

2 R. Swaminathan, Clin. Biochem. Rev., 2003, 24, 47-66.

3 R. J. Elin, Magnesium Res., 2010, 23, 194-198.

4 U. Gröber, J. Schmidt and K. Kisters, Nutrients, 2015, 7, 8199-8226.

5 S. M. Al-Ghamdi, E. C. Cameron and R. A. Sutton, Am. J. Kidney Dis., 1994, 24, 737-752.

6 Z. S. Agus, J. Am. Soc. Nephrol., 1999, 10, 1616-1622.

7 K. J. Martin, E. A. Gonzalez and E. Slatopolsky, J. Am. Soc. Nephrol., 2009, 20, 2291-2295.
8 Y. Sakaguchi, N. Fujii, T. Shoji, T. Hayashi, H. Rakugi and Y. Isaka, Kidney Int., 2013, 85, 174-181.

9 M. P. Staiger, A. M. Pietak, J. Huadmai and G. Dias, Biomaterials, 2006, 27, 1728-1734.

10 A. C. Daly, L. Riley, T. Segura and J. A. Burdick, Nat. Rev. Mater., 2020, 5, 20-43.

11 Y. S. Zhang and A. Khademhosseini, Science, 2017, 356, eaaf3627.

12 J. D. Tang, C. Mura and K. J. Lampe, J. Am. Chem. Soc., 2019, 141, 4886-4899.

13 L. Li, B. Xiao, J. Mu, Y. Zhang, C. Zhang, H. Cao, R. Chen, H. K. Patra, B. Yang, S. Feng, Y. Tabata, N. K. H. Slater, J. Tang, Y. Shen and J. Gao, ACS Nano, 2019, 13, 14283-14293.

14 J. Li and D. J. Mooney, Nat. Rev. Mater., 2016, 1, 16071.

15 J. H. Lee, A. El-Fiqi, N. Mandakhbayar, H. H. Lee and H. W. Kim, Biomaterials, 2017, 142, 62-76.

$16 \mathrm{C} . \mathrm{Wu}$ and J. Chang, J. Controlled Release, 2014, 193, 282-295.

17 K. Boldbaatar, K. Dashnyam, J. C. Knowles, H. H. Lee, J. H. Lee and H. W. Kim, Acta Biomater., 2019, 83, 322-333.

18 G. Z. Tan, P. E. Orndorff and R. A. Shirwaiker, J. Med. Biol. Eng., 2019, 39, 622-631.

19 A. M. El-Kady, A. A. Ali and A. El-Fiqi, J. Non Cryst. Solids, 2020, 534, 119889.

20 L. A. Sharpe, A. M. Daily, S. D. Horava and N. A. Peppas, Expert Opin. Drug Delivery, 2014, 11, 901-915.

21 M. C. Koetting, J. F. Guido, M. Gupta, A. Zhang and N. A. Peppas, J. Controlled Release, 2016, 221, 18-25.

22 C. A. Schoener, H. N. Hutson and N. A. Peppas, J. Biomed. Mater. Res., Part A, 2013, 101, 2229-2236.

23 J. F. Pinto, Int. J. Pharm., 2010, 395, 44-52.

24 S. Wang, Y. Ha, X. Huang, B. Chin, W. Sim and R. Chen, ACS Appl. Mater. Interfaces, 2018, 10, 36622-36627.

25 L. Liu, W. Yao, Y. Rao, X. Lu and J. Gao, Drug Delivery, 2017, 24, 569-581.

26 D. Lalezari, Ann. Gastroenterol. Hepatol., 2012, 25, 333-337.

27 J. Fallingborg, Dan. Med. Bull., 1999, 46, 183-196.

28 S. Wang, X. Liu, I. J. Villar-Garcia and R. Chen, Macromol. Biosci., 2016, 16, 1258-1264.

29 R. Chen, Z. Yue, M. E. Eccleston, S. Williams and N. K. H. Slater, J. Controlled Release, 2005, 108, 63-72.

30 R. Chen, S. Khormaee, M. E. Eccleston and N. K. H. Slater, Biomaterials, 2009, 30, 1954-1961.

31 Organization for economic cooperation and development, 2002. Screening information data set for isophthalic acid. IPCS INCHEM, Paris, France.

32 S. Wang, R. Attah, J. Li, Y. Chen and R. Chen, ACS Biomater. Sci. Eng., 2018, 4, 4236-4243.

33 K. A. Watkins and R. Chen, Int. J. Pharm., 2015, 478, 496-503.

34 K. Zhang, Z. Jia, B. Yang, Q. Feng, X. Xu, W. Yuan, X. Li, X. Chen, L. Duan, D. Wang and L. Bian, Adv. Sci., 2018, 5, 1800875.

35 K. Zhang, Q. Feng, J. Xu, X. Xu, F. Tian, K. W. Yeung and L. Bian, Adv. Funct. Mater., 2017, 27, 1701642. 
36 Y. J. Li, X. B. Hu, X. L. Lu, D. H. Liao, T. T. Tang, J. Y. Wu and D. X. Xiang, Drug Delivery, 2017, 24, 1868-1873.

37 K. O. Aboalnaja, S. Yaghmoor, T. A. Kumosani and D. J. Mcclements, Expert Opin. Drug Delivery, 2016, 13, 1327-1336.

38 S. Khani, F. Keyhanfar and A. Amani, Drug Delivery, 2016, 23, 2035-2043.

39 X. Gao, Y. Cao, X. Song, Z. Zhang, X. Zhuang, C. He and X. Chen, Macromol. Biosci., 2014, 14, 565-575.

40 R. Chen, M. E. Eccleston, Z. Yue and N. K. H. Slater, J. Mater. Chem., 2009, 19, 4217-4224.

41 R. Chen, S. Khormaee, M. E. Eccleston and N. K. Slater, Biomacromolecules, 2009, 10, 2601-2608.

42 A. Gupta, H. B. Eral, T. A. Hatton and P. S. Doyle, Soft Matter, 2016, 12, 2826-2841.

43 H. H. Tayeb and F. Sainsbury, Nanomedicine, 2018, 13, 2507-2525.
44 S. H. Hosseini and E. Forssberg, Min. Metall. Explor., 2006, 23, 87-96.

45 S. F. Pang, C. Q. Wu, Q. N. Zhang and Y. H. Zhang, J. Mol. Struct., 2015, 1087, 46-50.

46 N. Wang, C. Cai, X. He, S. F. Pang and Y. H. Zhang, Spectrochim. Acta, Part A, 2018, 192, 420-426.

47 J. Jahnen-Dechent and M. Ketteler, Clin. Kidney J., 2012, 5, i3-i14.

48 L. Salvia-Trujillo, Nanomaterials, 2016, 6, 17.

49 D. J. McClements, Soft Matter, 2011, 7, 2297-2316.

50 L. Salvia-Trujillo, C. Qian, O. Martín-Belloso and D. J. McClements, Food Chem., 2013, 141, 1472-1480.

51 H. Toguchi, Y. Ogawa and T. Shimamoto, Chem. Pharm. Bull., 1990, 38, 2797-2800.

52 L. Zou, B. Zheng, W. Liu, C. Liu, H. Xiao and D. J. Mcclements, J. Funct. Foods, 2015, 15, 72-83. 